\title{
Quaternionic Dirac Scattering
}

\section{De Leo $\mathbf{S}^{1 *}$, Ducati $\mathbf{G}^{2}$ and Giardino $\mathbf{S}^{3}$}

${ }^{1}$ Department of Applied Mathematics, State University of Campinas, Brazil

${ }^{2} \mathrm{CMCC}$, Universidade Federal do ABC, S̃ao Paulo, Brazil

${ }^{3}$ Department of Applied Mathematics, State University of Campinas, Brazil

\begin{abstract}
The scattering of a Dirac particle has been studied for a quaternionic potential step. In the potential region an additional diffusion solution is obtained. The quaternionic solution which generalizes the complex one presents an amplification of the reflection and transmission rates. A detailed analysis of the quaternionic spinorial velocities shed new light on the additional solution. For pure quaternionic potentials, the interesting and surprising result of total transmission is found. This suggests that the presence of pure quaternionic potentials cannot be seen by analyzing the reflection or transmission rates. It has been observed by measuring the mean value of some operator.
\end{abstract}

Keywords: Quaternions; Dirac equation; Scattering

\section{Introduction}

There are several proposals to formulate quantum mechanics $[1,2]$ by generalizing the complex number field. The most obvious generalization is done by introducing quaternions [3-20], but there are other possibilities, like Clifford algebras [21-25] and p-adic numbers [26]. Even inside each proposal, there may be internal divisions. In this article, we study a quaternionic quantum mechanics endowed with a quaternionic scalar product [3]. Our interest in the quaternionic formulation of Dirac equation is motivated by the recent interesting results obtained in the standard Dirac theory for the diffusion [2730], tunneling [31-33] and Klein zones [34-38]. The generalization to quaternionic potentials [20] could be useful to understand many hidden aspects of the Dirac solutions. Even simple solutions, as those involving the potential step, contain interesting results. In the case of quaternionic quantum mechanics, there are fewer studies which investigate the quaternionic formulation of Dirac equation. In Adler's book [3], the theoretical foundations have been laid down, nevertheless explicit physical examples are still quite rare. More recently, the Dirac solutions in the presence of quaternionic potentials have been detailed discussed in ref. [20]. In the potential region, the quaternionic solutions allow two values for the wave-function momentum. This represents the first important difference with respect to the complex case in which only one value is found. A better understanding of the additional quaternionic solution can be achieved by studying an explicit example of quaternionic potential and calculating the reflection and transmission coefficients. In order to do this, we recall the general solution presented in a previous article [20] and study the scattering by a quaternionic potential step. In the complex scattering, for one-dimensional motion, we have not spin flip in the reflected and transmitted beams $[2,29,30]$. Thus, the spin flip is not considered too for the quaternionic case. The additional quaternionic solutions and the new reflection and transmission coefficients satisfy conservation of probability and in the complex limit reproduce the standard Dirac scattering. A novel and intriguing behavior is observed for the scattering by a purely quaternionic step. In this case, there is no reflection and the wave-function shows an oscillating spatial pattern similar to the one observed for circularly polarized light. The article is organized as follows. In section II, for the convenience of the reader we fix our notation and recall the Dirac solution in the presence of a quaternionic potential. In section III, we obtain the reflection and transmission coefficients and calculate the probability current. In section IV, we discuss the effect of the quaternionic potential on the particle velocities.
The last sections round off the article with a brief discussion on the total transmission for pure quaternionic potentials (section V), final considerations and future possible investigations (section VI).

\section{The Quaternionic Dirac Equation}

As usual in quaternionic quantum mechanics [3], the Dirac equation is written in terms of an anti- hermitian Hamiltonian operator. In this spirit, we introduce the anti-hermitian quaternionic potential step

$\left\{0\right.$ for $z<0$ (REGION I), $i V_{0}+j V_{1}+k V_{2}$ for $z>0$ (REGION II) $\}$

Where $V_{0,1,2}$ are real constants. In the potential region (region II), the Dirac equation then reads

$$
\partial_{t} \Psi(r, t)=-\left[\alpha . \nabla+i m \beta+i V_{0}+j V_{1}+k V_{2}\right] \Psi(r, t)
$$

For $V_{1}=V_{2}=0$, the complex step is recovered. The Dirac Hamiltonian conatins the matrices $\alpha$ and $\beta$ which satisfy the algebra

$$
\alpha=\alpha^{\dagger}, \beta=\beta^{\dagger}, \alpha^{2}=\beta^{2}=1,\{\beta, \alpha\}=0 \text { and }\left\{\alpha_{r}, \alpha_{r}\right\}=2 \delta_{r s} 1
$$

In the Dirac representation [2], we have

$$
\alpha=\left(\begin{array}{cc}
0 & \sigma \\
\sigma & 0
\end{array}\right), \beta=\left(\begin{array}{cc}
1 & 0 \\
0 & -1
\end{array}\right)
$$

Where $\sigma$ are the well-known complex Pauli matrices. For convenience of notation, the quaternionic Potential can be rewritten as follows

$$
W_{0}=V_{2}+i V_{1}=\left|W_{0}\right| \exp \left[\mathrm{i} \arctan \left(V_{1} / V_{2}\right)\right]=\left|W_{0}\right| \exp [i \phi] .
$$

Considering a motion along the $\mathrm{z}$-axis, $\mathrm{p}=(0,0, \mathrm{Q})$, introducing the quaternionic spinor

$$
\Psi(r, t)=[u+j w] \exp \left[i\left(Q_{z}-E t\right)\right]
$$

*Corresponding author: Stefano De Leo, Department of Applied Mathematics, State University of Campinas, Brazil, Tel: 5519 352159; E-mail: deleo@ime.unicamp.br

Received November 28, 2014; Accepted February 17, 2015; Published March 10 2015

Citation: De Leo S, Ducati G, Giardino S (2015) Quaternionic Dirac Scattering. J Phys Math 6: 130. doi:10.4172/2090-0902.1000130

Copyright: $\odot 2015$ De Leo S, et al. This is an open-access article distributed under the terms of the Creative Commons Attribution License, which permits unrestricted use, distribution, and reproduction in any medium, provided the original author and source are credited. 
Where $\mathrm{u}$ and $\mathrm{v}$ are complex spinors, we obtain two coupled complex matrix equations,

$$
\begin{aligned}
& \left(E-Q \alpha_{3}-m \beta-V_{0}\right) u=-W_{0}^{*} w \\
& \left(E-Q \alpha_{3}-m \beta+V_{0}\right) w=-W_{0}^{*} u
\end{aligned}
$$

The first equation is obtained from the complex part of equation (1), and the second one is obtained taking the pure quaternionic part. Equations (5) can be rewritten as eigenvalue equations,

$$
\begin{aligned}
& \left(E-Q \alpha_{3}+m \beta+V_{0}\right)\left(E-Q \alpha_{3}-m \beta-V_{0}\right) u=\left|W_{0}\right|^{2} u \\
& \left(E-Q \alpha_{3}-m \beta-V_{0}\right)\left(E-Q \alpha_{3}+m \beta+V_{0}\right) u=\left|W_{0}\right|^{2} w
\end{aligned}
$$

The determinants of the matrices on the left hand side of equations (6) are equal, and consequently the eigenvalues obtained from any of them are also equal. Non-trivial solutions have to satisfy

$$
\operatorname{det}\left[\left(E-Q \alpha_{3}+m \beta+V_{0}\right)\left(E-Q \alpha_{3}-m \beta-V_{0}\right)-\left|W_{0}\right|^{2}\right]=0
$$

From equation (7), we obtain two squared momenta for particles moving in the region II, namely

$$
Q_{ \pm}^{2}=q_{ \pm}^{2}+\left|W_{0}\right|^{2} \pm 2 \delta
$$

Where

$$
q_{ \pm}^{2}=\left(E \pm V_{0}\right)^{2}-m^{2}, \delta=\sqrt{\left(E V_{0}\right)^{2}+\left(p\left|W_{0}\right|\right)^{2}-} E V_{0} \text { and } p=\sqrt{E^{2}-m^{2}}
$$

From equation (8), $Q_{+}^{2}>0$ and $Q_{-}^{2}$ may be either positive or negative. Hence, we find three energy zones determined by the $Q_{-}^{2}$ value,

Diffusion zone $Q_{-}^{2}>0$ and $E>\sqrt{\left|W_{0}\right|^{2}+\left(V_{0}-m\right)^{2}}$,

Tunnelingzone $Q_{-}^{2}<0$ and, $\max \left[m, \sqrt{\left|W_{0}\right|^{2}+\left(V_{0}-m\right)^{2}}\right]<E<\sqrt{\left|W_{0}\right|^{2}+\left(V_{0}+m\right)^{2}}$

Klein zone $Q_{-}^{2}>0$ and $E<\sqrt{\left|W_{0}\right|^{2}+\left(V_{0}-m\right)^{2}}$.

These regions generalize the diffusion, tunneling, and Klein zones obtained for the complex case [27-38],

1) Diffusion zone $q_{-}^{2}>0$ and $E>V_{0}+m$

2) Tunneling zone $q_{-}^{2}<0$ and $V_{0}-m<E<V_{0}+m$,

3) Klein zone $q_{-}^{2}>0$ and $E<V_{0}-m$

For diffusion [27-30] and Klein [34-38] zones, we find oscillatory solutions interpreted as particles or anti-particles. In the tunneling zone [31-33] the wave function becomes evanescent and thus we have not particle or anti-particle propagation. In order to explicitly determine the quaternionic wave functions, we calculate the eigenvectors of equations (6). The four component eigenvectors can be conveniently written by defining the two- dimensional vectors

$\chi=\left\{(1,0)^{t},(0,1)^{t}\right\}$. After simple algebraic manipulation, for, we find

$u=\left[\frac{\chi}{E-V_{0}+m-\frac{\delta}{E-m}} \sigma_{3} \chi\right]$ and $w=-W_{0}\left(E-Q_{-} \alpha_{3}+m \beta+V_{0}\right)^{-1} u$,

And for $Q=Q_{+}$,

$$
\tilde{w}=\left[\begin{array}{c}
\frac{Q_{+}}{E-V_{0}+m+\frac{\delta}{E-m}} \sigma_{3} \chi \\
\chi
\end{array}\right] \text { and } \tilde{u}=-W_{0}^{*}\left(E-Q_{+} \alpha_{3}-m \beta-V_{0}\right)^{-1} \tilde{w} .
$$

\section{The Matching Conditions}

In order to study the scattering of a Dirac particle by the quaternionic step, for simplicity of calculation we choose a spin orientation for the incoming particle, i.e. $\chi=(10)^{t}$ and explicit in the complex spinors $u, w, \tilde{u}$ and $\tilde{w}$ their dependence on $Q, V_{0}$ and $W_{0}$. For example, in region $\mathrm{I}$, we have an incident particle with momentum $p=\sqrt{E^{2}-m^{2}}$ thus represented by $u[-p ; 0,0]$. There is no spin flip in the reflection or transmission, thus we can eliminate the zero component in our wave functions and work with two-component quaternionic spinors. In region I (potential free region), the quaternionic wave function is then given by

$$
\Psi_{\mathrm{I}}(z)=u[p ; 0,0] \exp [i p z]+[u[-p ; 0,0] R+j \tilde{w}[-p ; 0,0] \tilde{R}] \exp [-i p z],
$$

where $\mathrm{R}$ and $\tilde{R}$ are complex coefficients. Note that the presence of complex exponential in (4) requires multiplication from the right by complex coefficients. In region II (potential region), the quaternionic wave function is characterized by two possible momenta, $Q_{+}$and $Q_{-}$, and its explicit expression is

$\Psi_{\mathrm{II}}(z)=\left[u\left[Q ; V_{0}, W_{0}\right]+j w\left[Q ; V_{0}, W_{0}\right]\right] T \exp [i Q, z]+\left[u\left[Q ; V_{0}, W_{0}\right]+j \tilde{w}\left[Q ; V_{0}, W_{0}\right]\right] \tilde{T} \exp [i Q z z]$

As happens for the reflection coefficients, the transmission coefficients $T$ and $\tilde{T}$ are also complex. The matching condition, $\Psi_{\mathrm{I}}(0)=\Psi_{\mathrm{II}}(0)$, implies

$$
\begin{aligned}
& u[p ; 0,0]+u[-p ; 0,0] R=u\left[Q_{-} ; V_{0}, W_{0}\right] T+\tilde{u}\left[Q_{+} ; V_{0}, W_{0}\right] \tilde{T} \\
& \tilde{w}[-p ; 0,0] \tilde{R}=w\left[Q_{-} ; V_{0}, W_{0}\right] T+\tilde{w}\left[Q_{+} ; V_{0}, W\right] \tilde{T}
\end{aligned}
$$

From these coupled equations, we can obtain the complex coefficients $R, \tilde{R}, T, \tilde{T}$ in terms of the wave function parameters and consequently calculate the transmission and reflection rates.

\section{The reflection and transmission coefficients}

To simplify our notation let us introduce the a dimensional quantity $a=\frac{p}{E+m}$ and explicitly rewrite the quaternionic wave function in region $\mathrm{I}$,

$$
\Psi_{\mathrm{I}}(z)=\left(\begin{array}{l}
1 \\
a
\end{array}\right) \exp [i p z]+\left(\begin{array}{c}
1 \\
-a
\end{array}\right) R \exp [-i p z]+j\left(\begin{array}{c}
-a \\
1
\end{array}\right) \tilde{R} \exp [-i p z],
$$

and region $\mathrm{II}$,

$$
\Psi_{\mathrm{II}}(z)=\left[\left(\begin{array}{c}
1 \\
A_{-}
\end{array}\right)-j W_{0}\left(\begin{array}{c}
M_{-} \\
N_{-}
\end{array}\right)\right] T \exp \left[i Q_{-} z\right]+\left[-W_{0}^{*}\left(\begin{array}{c}
N_{+} \\
M_{+}
\end{array}\right)+j\left(\begin{array}{c}
A_{+} \\
1
\end{array}\right)\right] \tilde{T} \exp \left[i Q_{+} z\right],
$$

where

$A_{ \pm}=\frac{Q_{ \pm}}{E \pm V_{0}+m \pm \frac{\delta}{E-m}}, M_{ \pm}=\frac{Q_{ \pm} A_{ \pm}+E-m \mp V_{0}}{q_{\mp}^{2}-Q_{ \pm}^{2}}, N \pm=\frac{\left(E+m \mp V_{0}\right) A \pm Q \pm}{q_{\mp}^{2}-Q_{ \pm}^{2}}$

The continuity equations at $\mathrm{z}=0$ lead to the following system

$$
\begin{aligned}
& \left(\begin{array}{c}
1 \\
a
\end{array}\right)+\left(\begin{array}{c}
1 \\
-a
\end{array}\right) R=\left(\begin{array}{c}
1 \\
A_{-}
\end{array}\right) T-W_{0}^{*}\left(\begin{array}{c}
N_{+} \\
M_{+}
\end{array}\right) \tilde{T}, \\
& \left(\begin{array}{c}
-a \\
1
\end{array}\right) \tilde{R}=-W_{0}\left(\begin{array}{c}
M_{-} \\
N_{-}
\end{array}\right) T+\left(\begin{array}{c}
A_{+} \\
1
\end{array}\right) \tilde{T} .
\end{aligned}
$$

After algebraic manipulations, we find 


$$
\begin{aligned}
& R=\frac{\left(a-A_{-}\right)\left(a+A_{+}\right)+\left|W_{0}\right|^{2}\left(M_{+}-a N_{+}\right)\left(M_{-}+a N_{-}\right)}{\left(a+A_{-}\right)\left(a+A_{+}\right)-\left|W_{0}\right|^{2}\left(M_{+}+a N_{+}\right)\left(M_{-}+a N_{-}\right)}, \\
& T=\frac{2 a\left(a+A_{+}\right)}{\left(a+A_{-}\right)\left(a+A_{+}\right)-\left|W_{0}\right|^{2}\left(M_{+}+a N_{+}\right)\left(M_{-}+a N_{-}\right)}, \\
& \tilde{R}=W_{0} \frac{M_{-}-A_{+} N_{-}}{a+A_{+}} T, \\
& \tilde{T}=W_{0} \frac{M_{-}+a N_{-}}{a+A_{+}} T .
\end{aligned}
$$

In the complex limit, $\mathrm{W} 0 \rightarrow 0$, we recover the standard reflection and transmission rates $[2], \tilde{R}_{c}=\tilde{T}=0$,

$$
\begin{aligned}
& \text { And } R_{c}=\frac{p\left(E-V_{0}+m\right)-q_{-}(E+m)}{p\left(E-V_{0}+m\right)+q_{-}(E+m)}, \\
& T_{c}=\frac{2 p\left(E-V_{0}+m\right)}{p\left(E-V_{0}+m\right)+q_{-}(E+m)} .
\end{aligned}
$$

\section{The Current Probability Density}

The relation between the reflection and transmission coefficients can be obtained by using the continuity equation

$$
\frac{\partial \rho}{\partial t}+\nabla . J=0
$$

Where $\rho=\Psi^{\dagger} \Psi$ and $J=\Psi^{\dagger} \alpha \Psi$

Observing that $\Psi^{\dagger} \Psi$ is independent of time and the spatial dependence of $\Psi$ is only on the $\mathrm{z}$-coordinate, we find

$$
\partial_{z} \Psi^{\dagger} \alpha_{3} \Psi=0
$$

The continuity of the current density between the free region I and the potential region II implies

$$
\Psi_{i n c}^{+} \alpha_{3} \Psi_{i n c}+\Psi_{R}^{+}+\Psi_{\bar{R}}^{+} \alpha_{3} \Psi_{\bar{R}}=\Psi_{T}^{+} \alpha_{3} \Psi_{\bar{T}} \alpha_{3} \Psi_{\bar{T}} .
$$

For the previous equation, we find

$$
|R|^{2}+|\tilde{R}|^{2}+\underbrace{\left(\frac{A_{-}+\left|W_{0}\right|^{2} M_{-}^{*} N_{-}}{2 a}+\text { h.c. }\right)}_{\rho}|T|^{2}+\underbrace{\left(\frac{A_{+}+\left|W_{0}\right|^{2} M_{+}^{*} N_{+}}{2 a}+\text { h.c. }\right)|\tilde{T}|^{2}=1}_{\tilde{\rho}} .
$$

In the complex limit, $\mathrm{W}_{0} \rightarrow 0$, we recover the well know continuity equation

$$
\left|R_{c}\right|^{2}+\underbrace{\frac{\left(q_{-}+q_{-}^{*}\right)(E+m)}{2 p\left(E-V_{0}+m\right)}\left|T_{c}\right|^{2}=1}_{\rho_{c}}
$$

For $E>V_{0}+m$, we have $q_{-} \in \mathbb{R}$ and $\rho_{c}>0$. Eq. (20) then implies a reflection rate $<1$ (diffusion zone). For $V_{0}-m<E<V_{0}+m$, we have $q_{-}^{2}<0$. Consequently, $\rho_{c}=0$ and Eq.(20) implies a reflection rate $=1$. Finally, for $E<V_{0}-m$, we have $q_{-} \in \mathbb{R}$ and $\rho_{c}<0$, we find a reflection rate $>1$ suggesting pair production [2,34-38]. In Figure 1, we plot the reflection rates for different value of quaternionic potentials as a function of $\mathrm{E} / \mathrm{m}$. In the Klein zone, the reflection rate increases by increasing the quaternionic potential. In this energy zone, the quaternionic perturbation thus contributes to the phenomenon of pair production. This is confirmed by the transmission rates in Figure 2.

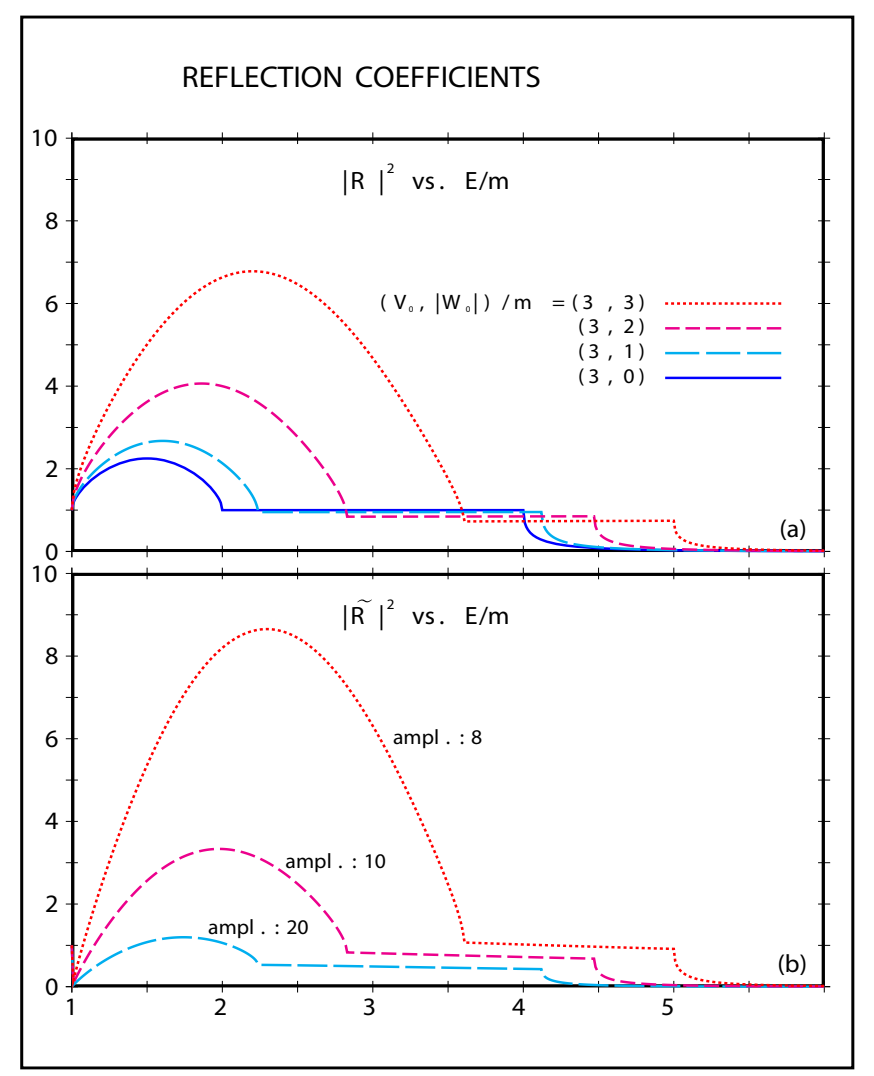

Figure 1: The reflection rates as a function of the incoming energy for a fixed complex potential $\mathrm{V} 0=3 \mathrm{~m}$ (blue continuous line) and for quaternionic perturbation $|\mathrm{W} 0|=(1,2,3) \mathrm{m}$ (cyan/magenta/red dashed and dotted lines). By increasing the quaternionic part of the potential, the tunneling zone decreases. We also observe that in presence of quaternionic perturbation, due to the additional diffusion solution, we have not total reflection in the tunneling zone.

It is important to observe that for complex tunneling only evanescent waves, $\exp \left[-\left|q_{-}\right| z\right]$, appears in the potential region and this is seen by observing (Figure 1a) that the reflection rate is 1 . The presence of a quaternionic potential breaks total reflection and this is explained by the fact that together evanescent wave, $\exp \left[-\left|Q_{-}\right| z\right]$, also oscillatory waves, $\exp \left[i\left|Q_{+}\right| z\right]$, are present in the potential region. The numerical calculation for the combined reflection and transmission coefficients is shown in Figure 3 and confirms the continuity equation (19). From Figure 3, we also observe a shift in the starting point of the diffusion zone and more important we have a decreasing energy zone for the evanescent waves by increasing the quaternionic potential. The last observation clearly suggest that oscillatory waves kill evanescent waves in presence of great quaternionic potentials. Finally, we also find that the reflection and transmission rates $|\tilde{R}|$ and $|\tilde{T}|$ are very small compared to the reflection rates $|R|$ and $|T|$, thus as a first approximation we can use these last coefficient rates to study quaternionic perturbations of complex quantum scattering problems.

\section{Group Velocities Analysis}

The incoming complex plane wave has the standard oscillatory exponential, $\exp [i(p z-E t)]$, and consequently its group velocity is given by 


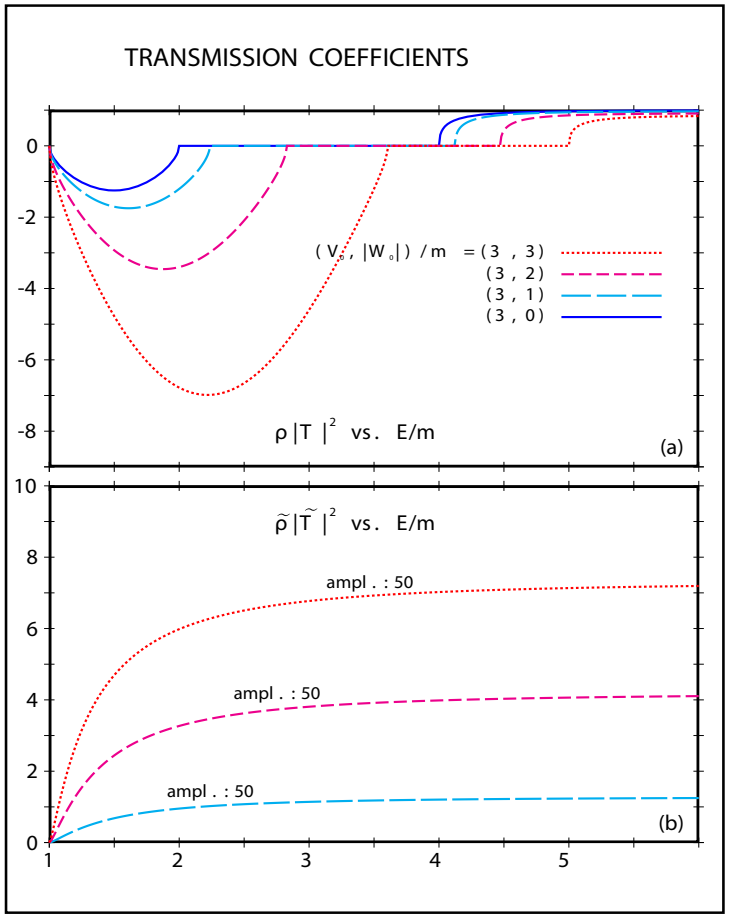

Figure 2: The transmission rates are plotted as function of the incoming energy for a fixed complex potential and different quaternionic perturbation. As observed in the caption of Figure 1, in presence of Quaternionic potentials total reflection breaks down. This is clear from the plot in (b) which confirms The presence of additional diffusion solutions.

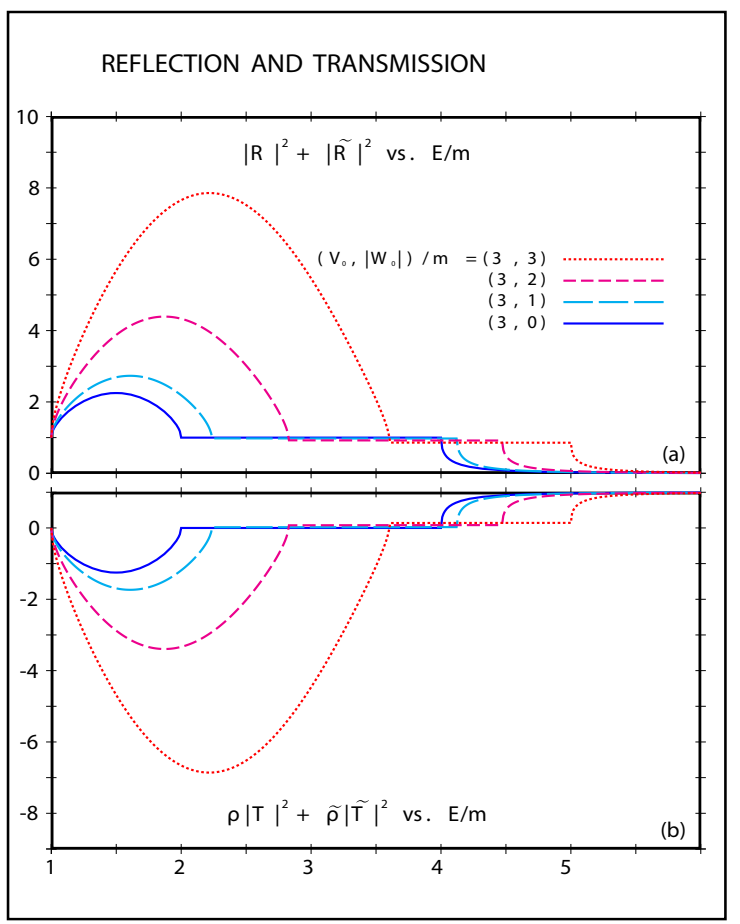

Figure 3: The reflection and transmission rates satisfy the probability conservation. In the Klein zone, we find a negative transmission flux and consequently a reflection rate greater than one. This effect is explained in terms of pair production. The quaternionic perturbation amplifies the pair production.

$$
v_{i n}=\frac{d E}{d p}=\frac{p}{E}
$$

The transmitted quaternionic plane waves contain, in the diffusion and Klein zones, the oscillatory exponentials $\exp \left[i\left(Q_{ \pm} z-E t\right)\right]$, which determine the following group velocities

$$
v_{ \pm}=\frac{d E}{d Q_{ \pm}} \text {. }
$$

Recalling that

$$
Q_{ \pm}^{2}=\left(E \pm V_{0}\right)^{2}-m^{2}+\left|W_{0}\right|^{2} \pm\left(\sqrt{E^{2}\left(V_{0}^{2}+\left|W_{0}\right|^{2}-m^{2}\left|W_{0}\right|^{2}-E V_{0}\right.}\right)
$$

We immediately obtain

$$
Q_{ \pm} d Q_{ \pm}=\left[E \pm V_{0} \pm \frac{E\left(V_{0}^{2}+\left|W_{0}\right|^{2}\right.}{\sqrt{E^{2} V_{0}^{2}+p^{2}\left|W_{0}\right|^{2}}} \mp V_{0}\right] d E \text {, }
$$

and consequently

$$
v_{ \pm}=\frac{Q_{ \pm}}{E}\left(1 \pm \frac{V_{0}^{2}+\left|W_{0}\right|^{2}}{\sqrt{E^{2} V_{0}^{2}+p^{2}\left|W_{0}\right|^{2}}}\right)^{-1}
$$

The additional quaternionic spinor is then characterized by a velocity $v_{+}$which is a typical velocity of diffusion Figure $4 \mathrm{a}$. Indeed, we have not tunneling or Klein zones as happens for the velocity $v_{-}$ Figure $4 \mathrm{~b}$. By increasing the quaternionic part of the potential we see decreasing the velocity of the additional quaternionic spinor and by

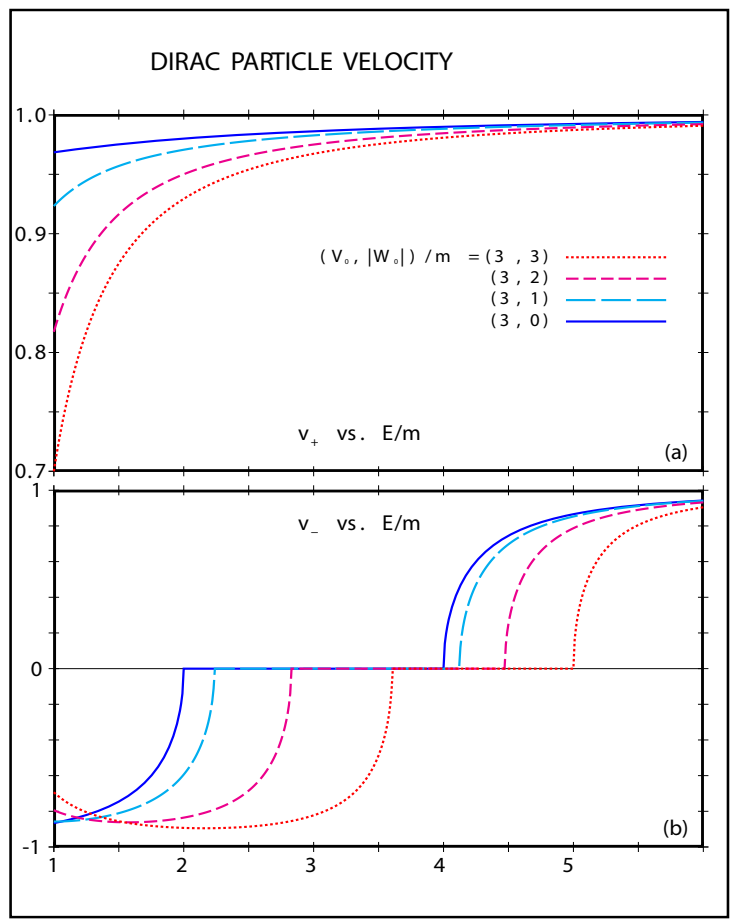

Figure 4: The group velocities, plotted as function of the incoming energy, confirms the analysis done for the reflection and transmission rates. The quaternionic perturbation acts in the diffusion zone by decelerating the particle and by decreasing the tunneling energy zone. 
increasing the incoming energy we obtain an increasing value of the velocity. These are typical effects of diffusion. As observed before, the velocity $v_{-}$presents three different energy zones (see Figure $4 \mathrm{~b}$ ), the diffusion zone for $E>\sqrt{|W|_{0}^{2}+\left(V_{0}+m\right)}$ where the potential acts decelerating the particles, the Klein zone for $E<\sqrt{|W|_{0}^{2}+\left(V_{0}-m\right)}$ where the negative velocities solutions generates a flux from the right to the left which gives a reflection rates greater than one and consequently interpreted in terms of pair production and finally the intermediate tunneling zone where we have not propagation due to the presence of evanescent solutions. It is interesting to observe that in the diffusion zone, we have two propagation regimes and consequently two different velocities. These different velocities permit to decouple the two quaternionic solutions. In the pure quaternionic limit $(\mathrm{V} 0 \rightarrow 0$ ), as we shall discuss in our conclusions, these velocities tend to the same value and this value is $\mathrm{p} / \mathrm{E}$ the free propagation velocity.

\section{Total Transmission}

Our analysis shows a surprising effect for pure quaternionic potentials, $\mathrm{V} 0=0$. In this case,

$$
Q_{ \pm}=p \pm\left|W_{0}\right|, A_{ \pm}=a, M_{ \pm}=\mp \frac{a}{\left|W_{0}\right|} \text { and } N_{ \pm}=\mp \frac{1}{\left|W_{0}\right|},
$$

and the continuity equation leads to the following system for the reflection and transmission coefficients,

$$
1 \pm R_{q}=T_{q}+T e^{-i \phi} \text { and } \pm \tilde{R}_{q}=-T_{q} e^{i \phi}+\tilde{T}_{q} .
$$

The solutions are immediately obtained by showing total transmission,

$$
R_{q}=\tilde{R}_{q}=0 \quad T_{q}=\frac{1}{2} \quad \tilde{T}_{q}=T_{q} e^{i \phi} .
$$

The transmitted wave-function for the pure quaternionic potential is then given by

$$
\begin{aligned}
& \Psi_{q, T}=\left[\left(\begin{array}{c}
1 \\
a
\end{array}\right) \cos \left(\left|W_{0}\right| z\right)-k\left(\begin{array}{l}
a \\
1
\end{array}\right) e^{i \phi} \sin \left(\left|W_{0}\right| z\right)\right] e^{i p z} \\
& =\left[\begin{array}{lll}
\cos \left(\left(\left|W_{0}\right| z\right)-k e^{i \phi}\right. & \sin \left(\left|W_{0}\right| z\right) \\
-k e^{i \phi} & \sin \left(\left|W_{0}\right| z\right) & \cos \left(\left|W_{0}\right| z\right)
\end{array}\right] \underbrace{\left(\begin{array}{c}
1 \\
a
\end{array}\right) e^{i p z}}_{\Psi_{c, i n c}}
\end{aligned}
$$

and propagates with group velocity $\mathrm{p} / \mathrm{E}$. Due to the fact that the transmitted quaternionic spinor satisfies $\Psi_{q, T}^{\dagger} \Psi_{q, T}=1$, and moves with the same velocity of the free incoming particle seems impossible to see the presence of pure quaternionic potentials. Nevertheless the problem of an apparent invisible presence of pure quaternionic potential can be solved by taking the mean value of operators. For example, the $\mathrm{z}$ component spin mean value for the complex wave function $\Psi_{c, \text { inc }}$ is given by

$$
\left\langle S_{z}\right\rangle_{c}=\frac{\Psi_{c, i n c}^{\dagger} S_{z} \Psi_{c, i n c}}{\Psi_{c, i n c}^{\dagger} \Psi_{c, i n c}}=\frac{1-a^{2}}{2\left(1+a^{2}\right)}=\frac{m}{2 E},
$$

which in the non-relativistic limit, $\mathrm{p}<<\mathrm{m}$, reproduces the well-known result $\left\langle S_{z}\right\rangle_{c}=1 / 2$. In thepresence of a pure quaternionic potential, we should find

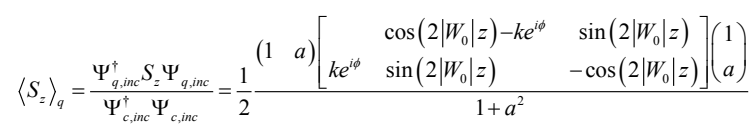

$$
=\frac{1-a^{2}}{2\left(1+a^{2}\right)} \cos \left(2\left|W_{0}\right| z\right)=\frac{m}{2 E} \cos \left(2\left|W_{0}\right| z\right)
$$

Which implies a z-dependence on the mean value of spin similar to what happens for circular polarized light $[39,40]$.

\section{Conclusions}

In this article we have studied the scattering of a Dirac particle by a quaternionic potential step. Our analysis shows the presence of an additional quaternionic solution characterized by a group diffusion velocity $v_{+}$. The quaternionic solution which generalizes the standard one is characterized by a group velocity $v_{-}$and presents three energy zones, diffusion-tunneling-Klein zones. For diffusion the two solutions are, in general, different and could be decouple the two solutions in the potential region showing the presence of a quaternionic potential. By increasing the quaternionic part of the potential we increase the Klein zone and decrease the tunneling zone, (Figure $4 \mathrm{~b}$ ). The most interesting effect is seen for a pure quaternionic potential where total transmission and a $\mathrm{z}$ dependence on the spin operators appear. This suggests a possible investigation in quaternionic optics. Before to do it, due to the fact that we have to introduce Gaussian laser, in a forthcoming, we aim to extend the plane wave analysis done in this article to quaternionic wave packets.

\section{References}

1. Cohen-Tannoudji C, Diu B, Laloe F (1997) Quantum mechanics. John-Wiley Sons, Paris.

2. Itzykson C, Zuber JB (1985) Quantum field theory, McGraw-Hill, Singapore.

3. Adler SL (1995) Quaternionic quantum mechanics and quantum fields, Oxford UP, New York.

4. Adler SL (1989) A new embedding of quantum electrodynamics in a nonabelian gauge structure. Phys Lett B 221: 39-43.

5. Davies AJ, McKellar BHJ (1989) Non relativistic quaternionic potentials in one dimension. Phys Rev A 40: 4209-4214.

6. Davies AJ (1990) Quater- nionic Dirac equation. Phys Rev D 41: 2628-2630

7. De Leo S, Rotelli P (1992) Quaternion scalar field. Phys Rev D 45: 575-579.

8. De Leo S, Rotelli P (1996) The quaternionic Dirac Lagrangian. Mod. Phys Lett A 11: 357-366.

9. De Leo S, Rotelli P (1996) Quaternionic electroweak theory. J Phys G 22 1137-1150.

10. De Leo S, Ducati G, Nishi C (2002) Quaternionic potentials in non-relativistic quantum mechanics. J Phys A 35: 5411-5426.

11. De Leo S, Ducati G (2005) Quaternionic bound states. J Phys A 38: 3443-3454

12. De Leo S, Ducati G, Madureira T (2006) Analytic plane wave solutions for the quaternionic potential step. J Math Phys 47: 082106-15.

13. De Leo S, Ducati G (2006) Quaternionic diffusion by a potential step. J Math Phys 47: 102104-9.

14. De Leo S, Ducati G (2007) Quaternionic wave packets. J Math Phys 48: 052111 10.

15. De Leo S, Ducati G, Leonardi, Pereira K (2010) A closed formula for the barrier transmission coefficient in quaternionic quantum mechanics. J Math Phys 51 113504-10.

16. LA Glinka and AW (2012) Beckwith, The Quaternionic Particle Mass, Prespacetime J 3: 126-130

17. De Leo S, Ducati G (2012) Delay time in quaternionic quantum mechanics. J Math Phys 53.

18. De Leo S, Ducati G (2013) The Snell law for quaternionic potentials. J Math Phys 54. 
19. De Leo S, Giardino S (2014) Dirac solutions for quaternionic potentials. J Math. Phys 55.

20. Hestenes D (1971) Vectors, spinors, and complex numbers in classical and quantum physics. Am J Phys. 39: 1013-1027.

21. Edmonds JD (1999) Dirac's equation in half of his algebra. Eur. J Phys 20: 461-467.

22. Hestenes D (2003) Space time physics with geometric algebra. Am J Phys 71: 691-714.

23. Rodrigues WA (2005) Algebraic and Dirac-Hestenes spinors and spinor fields. $J$ Math Phys 45: 2928-2944.

24. Hiley BJ (2012) Process, distinction, groupoids and Clibord algebras: an alternative view of the quantum formalism. Lect Notes Phys 813: 705-752.

25. Brekke L, Freund PGO (1993) p-adic numbers in physics. Phy Rept 233: 1-66.

26. De Leo S, Rotelli $P$ (2006) Above barrier Dirac multiple scattering and resonances. Eur Phys J 46: 551-558.

27. De Leo S Rotelli P (2009) Potential scattering in Dirac field theory. Eur Phys. J 62: 793-797.

28. De Leo S, Rotelli P (2009) Planar Dirac diffusion. Eur Phys J 63: 157-162.
29. De Leo S, Rotelli P (2012) Relative helicity phases in planar Dirac scattering Phys Rev A 86.

30. De Leo S, Rotelli P (2007) Dirac equation studies in the tunneling energy zone Eur Phys J C 51: 241-247.

31. De Leo S, Leonardi $V$ (2011) A new phase time formula for opaque barrier tunneling. J Phys A 4.

32. De Leo S, Leonardi V (2011) Relativistic tunneling through opaque barriers. Phys Rev A 83.

33. Klein O (1929) Die Reflexion von Elektronrn an einem Potentialsprun nach der relativistichen Dynamik von Dirac. Z Phys 53: 157-165.

34. Holstein BR (1998) Kleins paradox. Am J Phys 66: 507-512.

35. Nitta H, Kudo T, Minowa $\mathrm{H}$ (1999) Motion of a wave packet in the Klein paradox. Am J Phys 67: 966-971.

36. Krekora P, Su Q, Grobe R (2004) Klein paradox in spatial and temporal resolution. Phys Rev Lett 92.

37. De Leo S, Rotelli P (2006) Barrier paradox in the Klein zone. Phys Rev A 73.

38. Born M Wolf E (1999) Principles of optics. Cambridge UP, Cambridge.

39. Saleh BEA, Teich MC (2007) Fundamentals of Photonics. Wiley and Sons, New Jersey. 